\title{
An Overview of the Current Situation of Degloving Skin and Soft-Tissue Injuries in China: A Retrospective Study of the Inpatients with DSTI from 2013 to 2018
}

\section{Shaoshuo Yu}

Second Military Medical University First Hospital: Changhai Hospital

He Fang

Second Military Medical University First Hospital: Changhai Hospital

Yao-nan Jiang

Second Military Medical University First Hospital: Changhai Hospital

Chen-qi Tang

Second Military Medical University First Hospital: Changhai Hospital

\section{Ying Shi}

China Standard Medical Information Research Center

\section{Lan-xia Gan}

China Standard Medical Information Research Center

\section{Hong-tai Tang}

Second Military Medical University First Hospital: Changhai Hospital

\section{Hai-bo Wang}

Sun Yat-sen University First Affiliated Hospital

\section{Yu Sun}

Second Military Medical University First Hospital: Changhai Hospital

\section{Zhaofan Xia ( $\nabla$ xiazhaofan_smmu@163.com )}

Changhai Hospital https://orcid.org/0000-0002-8192-2693

\section{Research article}

Keywords: Degloving skin and soft-tissue injury, China, injury characteristics, treatment, epidemiological analysis

Posted Date: July 28th, 2021

DOl: https://doi.org/10.21203/rs.3.rs-715412/v1 
License: (c) (i) This work is licensed under a Creative Commons Attribution 4.0 International License. Read Full License 


\section{An Overview of the Current Situation of Degloving Skin and Soft-Tissue Injuries}

in China: A Retrospective Study of the Inpatients with DSTI from 2013 to 2018

Shao-shuo Yu ${ }^{1}$, He Fang ${ }^{1}$, Yao-nan Jiang ${ }^{1,2}$, Chen-qi Tang ${ }^{1}$, Ying Shi ${ }^{3}$, Lan-xia Gan ${ }^{3}$,

Hong-tai Tang ${ }^{1}$, Hai-bo Wang ${ }^{4 *}$, Yu Sun ${ }^{1 *}$ and Zhao-fan Xia ${ }^{1 *}$

${ }^{1}$ Department of Burn Surgery, the First Affiliated Hospital of Naval Medical University, Burn Institute of PLA, Research Unit of key techniques for treatment of burns and combined burns and trauma injury, Chinese Academy of Medical Sciences, Shanghai, P.R. China,

${ }^{2}$ The 92493 Hospital of the Chinese People's Liberation Army, Huludao, P.R. China,

${ }^{3}$ China Standard Medical Information Research Centre, Shenzhen, P.R. China,

${ }^{4}$ Clinical Trial Unit, First Affiliated Hospital of Sun Yat-Sen University, Guangzhou, P.R. China; Peking University, Centre for Data Science in Health and Medicine, Beijing, P.R. China

\section{*Corresponding author}

Zhaofan Xia, Department of Burn Surgery, the First Affiliated Hospital of Naval Medical University, Burn Institute of PLA, Research Unit of key techniques for treatment of burns and combined burns and trauma injury, Chinese Academy of Medical Sciences, 168 Changhai Road, Shanghai, P. R. China.

E-mail address: xiazhaofan@163.com

Yu Sun, Department of Burn Surgery, the First Affiliated Hospital of Naval Medical University, Burn Institute of PLA, Research Unit of key techniques for treatment of burns and combined burns and trauma injury, Chinese Academy of Medical Sciences, 
168 Changhai Road, Shanghai, P. R. China.

E-mail: littlefish0916@126.com

Haibo Wang, Clinical Trial Unit, First Affiliated Hospital of Sun Yat-Sen University,

Guangzhou, P. R. China; Peking University, Centre for Data Science in Health and Medicine, Beijing, P. R. China.

E-mail: haibo@mail.harvard.edu 


\begin{abstract}
Background: Degloving skin and soft-tissue injury (DSTI) is a kind of serious lesion in the field of surgery, with potential risk of morbidity and mortality. This study is aimed to summarize and analyze the current situation of DSTI in China, so as to provide enlightenments for better prevention and treatment.
\end{abstract}

Methods: By searching inpatients' information on the first page recorded by the Hospital Quality Monitoring System from January 1, 2013 to December 31, 2018, patients diagnosed with DSTI were identified and screened based on the International Classification of Diseases coding System. Demographic characteristics, injury and cost information were collected for analysis.

Results: There were 62709 patients who were diagnosed with DSTI, of whom $67.41 \%$ were males with a mean age of $43.01 \pm 19.70$. Peasants and workers, traffic-related accidents and falls, summer and autumn accounted for high percentage of the study indicators. The operation rate of DSTI roughly showed a growing trend, and the average length of stay was $22.02 \pm 29.73$ days, during which time $0.93 \%$ of the patients ended up in death. Medicine took up the first place of the hospitalization expense, but was decreasing year by year, while the proportion of other expenses gradually increased. More than half of the patients paid at their own charge, but the ratio of urban medical insurance was rising. The most and least frequent anatomic site of DSTI were lower extremity (43.40\%) and torso (1.59\%). Each injury site showed its own characteristics. Conclusions: This is the first retrospective study that targeted a nationwide data bank to make a relatively detailed epidemiological analysis of DSTIs from 2013 to 2018 in 
China. From this work, not only a preliminary understanding but also enlightenments for better prevention and treatment of DSTI has been gained.

Keywords: Degloving skin and soft-tissue injury, China, injury characteristics, treatment, epidemiological analysis 


\section{Background}

Degloving skin and soft-tissue injury (DSTI) is a kind of serious lesion in the field of surgery. The injured skin and the underlying tissue can peel off from the subcutaneous fascia or muscle layer under a sudden strong shear force(1), sometimes accompanied by extensive damage to inside structures such as muscle, blood vessels, nerves and bones(2). Patients with severe DSTI may suffer from complications like shock or infection, which can prolong the length of treatment and pose risk of disability or death(3), rendering great losses to themselves and their families. Therefore, it is very important to find efficacious therapies for DSTI in traumatology and other pertinent subjects. However, as nationwide investigation on DSTI remains a blank space, there is a lack of comprehensive understanding of the overall characteristics of this injury, which poses a severe challenge to clinical diagnosis and treatment. This study is aimed to summarize and analyze the demographic characteristics, injury situation, as well as cost information of patients with DSTI in China from 2013 to 2018, so as to provide enlightenments for better prevention and treatment.

\section{Methods}

By searching inpatients' information on the first page recorded by the Hospital Quality Monitoring System (HQMS) from January 1, 2013 to December 31, 2018, patients with DSTI were identified and screened based on the International Classification of Diseases (ICD-10) coding System. Demographic characteristics (gender, age, occupation, district, province), injury situation (admission way and season, 
injury site, injury factor, comorbidities), treatment (operation rate, operation type, operation frequency, length of stay, complications, nosocomial death) and cost information (overall expense, cost type, mode of payment) were collected for analysis, of which some in-hospital treatment-related indicators (the rate of operation, infection, shock, transfusion, ICU occupancy, nosocomial death, as well as LOS and hospitalization expense) were compared among different injury sites.

\section{Results}

There were 62709 patients who were diagnosed with DTSI, of whom $67.41 \%$ were males with a mean age of $43.01 \pm 19.70$. Demographics are presented in Figure 1. The majority were peasants (30.14\%) and workers (12.47\%). Among them, East China ranked the first $(28.54 \%)$ geographically, with Hubei province at the top (11.45\%).

Patients with DSTI were hospitalized through emergency $(58.79 \%)$, and tended to increase in summer and autumn (Fig 2.A), with the majority concentrated in July and August (20.91\%). Traffic-related accidents was the leading mechanism of injury (40.62\%), followed by falls (22.31\%) (Fig 2.B). According to the diagnostic codes from ICD coding system, DSTI of different anatomic sites were classified into four groups, namely, head (DSTI of the scalp), torso (DSTI of the lower back), upper extremity (including DSTI of the upper arms, forearms and hands) and lower extremity (including DSTI of the thighs, calves and feet). The most frequent anatomic site of DSTI was lower extremity (43.40\%), followed by head (31.33\%), upper extremity $(24.19 \%)$ and torso (1.59\%) (Fig 2.C). Comorbidities were found in $8.34 \%$ of all the inpatients, with 
hypertension being the most common (5.03\%), followed by diabetes $(2.15 \%)$ (Fig 2.D).

The operation rate of DSTI roughly showed a growing trend year by year (Fig 3.A), with a mean of $73.30 \%$, of which $37.09 \%$ were related to skin or flap transplanting and $31.93 \%$ underwent multi-operations. The average length of stay was $22.02 \pm 29.73$ days, during which time $0.93 \%$ of the patients ended up in death, and $12.13 \%$ developed complications, including infection (5.57\%) and shock (3.76\%), which happened far more often than the others (Fig 3.B).

The average hospitalization expense of DSTI patients was $33.51 \pm 53.59$ thousand RMB yuan. Medicine and consumables accounted for the largest proportion (59.57\%), but the former was decreasing markedly year by year, while growing trends emerged to varying degrees respectively among the rates of surgery, operation, nursing and examination expenses (Fig 4.A). More than half of the patients paid at their own charge $(51.10 \%)$, which proportion was rising together with that from by urban medical insurance, and those supported by the New Rural Cooperative Medical System had gradually decreased in recent years (Fig 4.B).

In-hospital treatment-related variables were then compared among the four different injury sites. Patients with the upper extremity DSTI seemed to have the best outcomes, who were more likely to undergo surgery, but had lower transfusion rate and ICU occupancy, as well as low incidence of complications like infection or shock, with significantly shorter LOS, less expenditure and lower nosocomial mortality. DSTI in the lower extremity needed more operations, with high transfusion rate, long hospital stay and correspondingly high cost. Though the incidence of DSTI in the torso was low, 
it showed high rate of shock and infection. Patients with head DSTI had the lowest operation rate, with the highest ICU occupancy and hospital mortality (Table 1).

\section{Discussion}

As the largest organ covering the surface of human body, our skin is the first protective barrier to resist external undesirable stimuli. It also has multiple functions such as excretion, temperature regulation and sensation(4), making it an important structure to maintain the internal stability of the organism. Although the incidence of DSTI is not high compared with other traumatic injuries, there is still a high risk of potential adverse consequences, because of the impairment of skin integrity and subcutaneous tissues. Rifat Latifi et al. proposed that the severity of DSTI mainly depends on the mechanism of injury, comorbidities (especially diabetes), concomitant injuries, injury site and type (open or closed)(3). Early identification of DSTI is essential, but the complex diversity of its clinical manifestations often makes this step intricate, leading to time-consuming and delayed treatment. Besides, there is no established guidelines or consensus on an appropriate treatment, therefore, timely and efficacious management of DSTI remains difficult(5). For many years, epidemiological investigation and detailed analysis have been carried out in this field(3-6), yet there is a paucity of similar large-scale research in China. This report is the first retrospective study that targeted a nationwide, multi-centric data bank to make a relatively detailed description of demographic and medical information of degloving skin and soft-tissue injuries during recent years in this country. Our study shows that the incidence of DSTI 
is $0.58 \%$ with a predilection to middle-aged males, and the leading mechanism of injury is traffic-related accidents, which is consistent with previous conclusions from similar studies in other countries $(5,7-9)$. Wearing clothing may be associated with the lower incidence of DSTI in winter season, as it decreases direct exposure of the skin(10). The number of rural patients (NRCBI) has been decreasing, while urban patients and those who can pay medical expenses at their own charge keep rising, which should be attributed to the acceleration of China's urbanization process and the improvement of people's economic level.

Current literature on DSTI is mostly based on specific anatomical sites. In this study, we found that the incidence of DSTI in lower extremities is the highest, somewhat in accordance with previous studies(3, 5, 11, 12). Notably, severe concomitant injuries could be the main lethal factors in some fatal cases(5). For instance, the rate of ICU occupancy and nosocomial mortality of DSTI in the head were higher than the other sites despite of its secondary incidence, which is owing to many traumatic brain injuries occurred along with DSTI, eliciting increased deaths. Although DSTI in torso was the least common, it had the highest complication rate of infection and shock. This result can be interpreted in two aspects. On the one hand, there is a good chance that patients diagnosed with DSTI of the torso also suffered from chest and abdominal viscera injuries, which caused heavy bleeding and increase the risk of infection. On the other hand, compared with head and limbs, the circulation of skin in torso is less sufficient, combined with the limitation of patients' position, which can make rehabilitation process harder. Of the four anatomical sites in our classification, DSTI of 
the upper extremity had the best treatment outcomes, with fewer complications and significantly shorter LOS, less expenditure and lower nosocomial mortality. Considering the indispensable functions of the upper extremity, particularly the hands, it is very reasonable to actively perform operations on them. Furthermore, the upper extremity is relatively small in area and rich in blood supply, plus the slightly less difficulty in postoperative nursing, so it has faster recovery and better prognosis.

The continuity of great vessels and capillary beds can be disrupted when DSTI occurs, followed by the formation of edema, which increase the diffusion distance of oxygen. The injured tissue swells within a confined space and becomes ischemic, creating a vicious cycle that leads to serious complications such as compartment syndrome(4). Necrosis of the skin can occur in the degloved area due to direct damage to the cutaneous layers or swelling of the subcutaneous tissue(13). These are generally considered to be serious complications of DSTI. Once they occur, the complexity and difficulty of treatment will increase dramatically. In this study, the incidence of skin necrosis and compartment syndrome was $0.87 \%$ and $0.30 \%$, respectively. Less than the proportion of infection and shock, though, it surpassed the concurrent rate of other complications, which should be noticed by surgeons. In addition to the routine prevention and treatment of common complications when managing DSTI, these two kinds of complications should be cautious about in case they occur and impair skin condition.

As this is the first time to carry out the big data analysis of DSTI, problems are inevitable. It is known that the trauma severity score (ISS/AIS)(5, 14, 15), which is 
widely used internationally to evaluate the severity of trauma on patients' first admission, has been a vital reference for guiding later treatment and predicting the prognosis. However, lack of such an item in HQMS makes it impossible for analysts to directly assess the severity of injury, instead they can only make predictions from indirect materials (length of stay, transfusion rate, ICU occupancy rate, complication rate, etc.), which affects the accuracy of final conclusions. If ISS/AIS could be added to the upgraded system subsequently, it would play a positive role in promoting more scientific and effective diagnosis and treatment of DSTI, even for all the trauma patients. Restricted by a variety of subjective and objective factors, there are inevitably some deviations in data screening, sorting and analysis in this study. On the premise of not distorting the final results, we've discarded the data that was incomplete or obviously divergent from the reality, and made recommendations to departments concerned on the current situation of non-standard information collection on the front page of medical records. Patients of different ages have their own characteristics in the clinical manifestations and prognosis of diseases due to the inherent disparities. By stratifying them according to their ages and making contrasts, we can expect a promising prospect when diagnosis and treatment of patients with trauma can be carried out in a more scientific way. More focus will be put on this in our future researches. As an injury of skin-related surgery, treatment of DSTI cannot be limited to a single subject(16). Irreversible damage to the skin caused by deep wounds means later troublesome problems such as the formation of scar, which hasn't yet been effectively resolved globally, and thus has long-term follow-up significance. This study is our first tentative 
exploration on the overview of DSTI in recent years in China. More in-depth investigation and discussion still need to be made in order to obtain a whole picture, and to provide more instructive conclusions for the prevention, diagnosis and treatment of DSTI as well.

\section{Conclusions}

This is the first retrospective study that targeted a nationwide data bank to make a relatively detailed epidemiological analysis of degloving skin and soft-tissue injuries from 2013 to 2018 in China. Through this work, we have not only obtained a preliminary understanding of the whole DSTI and the characteristics of each anatomical site, but also significant enlightenments for better prevention and treatment, as well as guidance to the future in-depth studies in this field. 


\section{List of abbreviations}

DSTI: Degloving Skin and Soft-Tissue Injury, HQMS: Hospital Quality Monitoring System, ICD: International Classification of Diseases, LOS: length of stay, ICU: intensive care unit, EC: East China, NC: North China, CC: central China, SC: South China, NWC: Northwest China, SWC: Southwest China, NEC: Northeast China, COPD: chronic obstructive pulmonary disease, CAD: coronary artery disease, CRF: chronic renal failure, DVT: deep venous thrombosis, GIB: gastrointestinal bleeding, UEBMI: Urban Employees' Basic Medical Insurance, URBMI: Urban Residents’ Basic Medical Insurance, NRCMI: New Rural Cooperative Medical Insurance, ISS/AIS: injury severity score/ abbreviated injury score 


\section{Declarations}

\section{Ethics approval and consent to participate}

According to the policy of the First Affiliated Hospital of Naval Medical University Ethics Committee, clinical data can be used and analyzed without revealing the identity of the patient. Since all the patient's identity information in HQMS was completely invisible to the analyzers, and all the analytical work was conducted on basis of secondary data, this study meets the basic requirements of the Declaration of Helsinki.

\section{Consent for publication}

Not applicable

\section{Availability of data and materials}

The data that support the findings of this study are available from China Standard Medical Information Research Centre but restrictions apply to the availability of these data, which were used under license for the current study, and so are not publicly available. Data are however available from the corresponding authors upon reasonable request and with permission of National Health and Development Commission.

\section{Competing interests}

The authors declare that they have no competing interests.

\section{Funding}

This work was funded by National Key R\&D Program of China (2019YFA0110600, 2019YFA0110602, 2019YFA0110603), National Natural Science Foundation of China (81930057, 81772076), CAMS Innovation Fund for Medical Sciences (2019-I2M-5-076), and Achievements Supportive Fund (2018-CGPZ-B03).

\section{Authors' contributions}

SSY, HF, YNJ, CQT, HTT and YS were involved in the conception and design of the study. SSY, HF and YNJ contributed to drafting the manuscript. SSY, LXG, YS and HBW participated in the data acquisition. All authors read and approved the final manuscript.

\section{Acknowledgements}

Not applicable 


\section{References}

1. Morris M, Schreiber MA, Ham B. Novel management of closed degloving injuries. J Trauma. 2009;67(4):E121-3.

2. Adani R, Marcoccio I, Castagnetti C, Tarallo L. Long-term results of replantation for complete ring avulsion amputations. Ann Plast Surg. 2003;51(6):564-8; discussion 9.

3. Latifi R, El-Hennawy H, El-Menyar A, Peralta R, Asim M, Consunji R, et al. The therapeutic challenges of degloving soft-tissue injuries. J Emerg Trauma Shock. 2014;7(3):228-32.

4. Antoniou D, Kyriakidis A, Zaharopoulos A, Moskoklaidis S. Degloving Injury.

5. Hakim S, Ahmed K, El-Menyar A, Jabbour G, Peralta R, Nabir S, et al. Patterns and management of degloving injuries: a single national level 1 trauma center experience. World J Emerg Surg. 2016;11:35.

6. Strazzieri-Pulido KC, Peres GR, Campanili TC, de Gouveia Santos VL. Incidence of Skin Tears and Risk Factors: A Systematic Literature Review. J Wound Ostomy Continence Nurs. 2017;44(1):29-33.

7. Mello DF, Assef JC, Soldá SC, Helene A, Jr. Degloving injuries of trunk and limbs: comparison of outcomes of early versus delayed assessment by the plastic surgery team. Rev Col Bras Cir. 2015;42(3):143-8.

8. Malone ML, Rozario N, Gavinski M, Goodwin J. The epidemiology of skin tears in the institutionalized elderly. J Am Geriatr Soc. 1991;39(6):591-5.

9. El-Menyar A, Consunji R, Asim M, Abdelrahman H, Zarour A, Parchani A, et al. Underutilization of occupant restraint systems in motor vehicle injury crashes: A quantitative analysis from Qatar. Traffic Inj Prev. 2016;17(3):284-91.

10. Kennedy P, Kerse N. Pretibial skin tears in older adults: a 2-year epidemiological study. J Am Geriatr Soc. 2011;59(8):1547-8.

11. Hudson DA, Knottenbelt JD, Krige JE. Closed degloving injuries: results following conservative surgery. Plast Reconstr Surg. 1992;89(5):853-5.

12. Milcheski DA, Ferreira MC, Nakamoto HA, Tuma P, Jr., Gemperli R. [Degloving injuries of lower extremity-- proposal of a treatment protocol]. Rev Col Bras Cir. 2010;37(3):199-203.

13. Nickerson TP, Zielinski MD, Jenkins DH, Schiller HJ. The Mayo Clinic experience with MorelLavallée lesions: establishment of a practice management guideline. J Trauma Acute Care Surg. 2014;76(2):493-7.

14. Low EE, Inkellis E, Morshed S. Complications and revision amputation following traumarelated lower limb loss. Injury. 2017;48(2):364-70.

15. Witt CE, Arbabi S, Nathens AB, Vavilala MS, Rivara FP. Obesity in pediatric trauma. J Pediatr Surg. 2017;52(4):628-32.

16. McGrouther DA, Sully L. Degloving injuries of the limbs: long-term review and management based on whole-body fluorescence. Br J Plast Surg. 1980;33(1):9-24. 
Figures
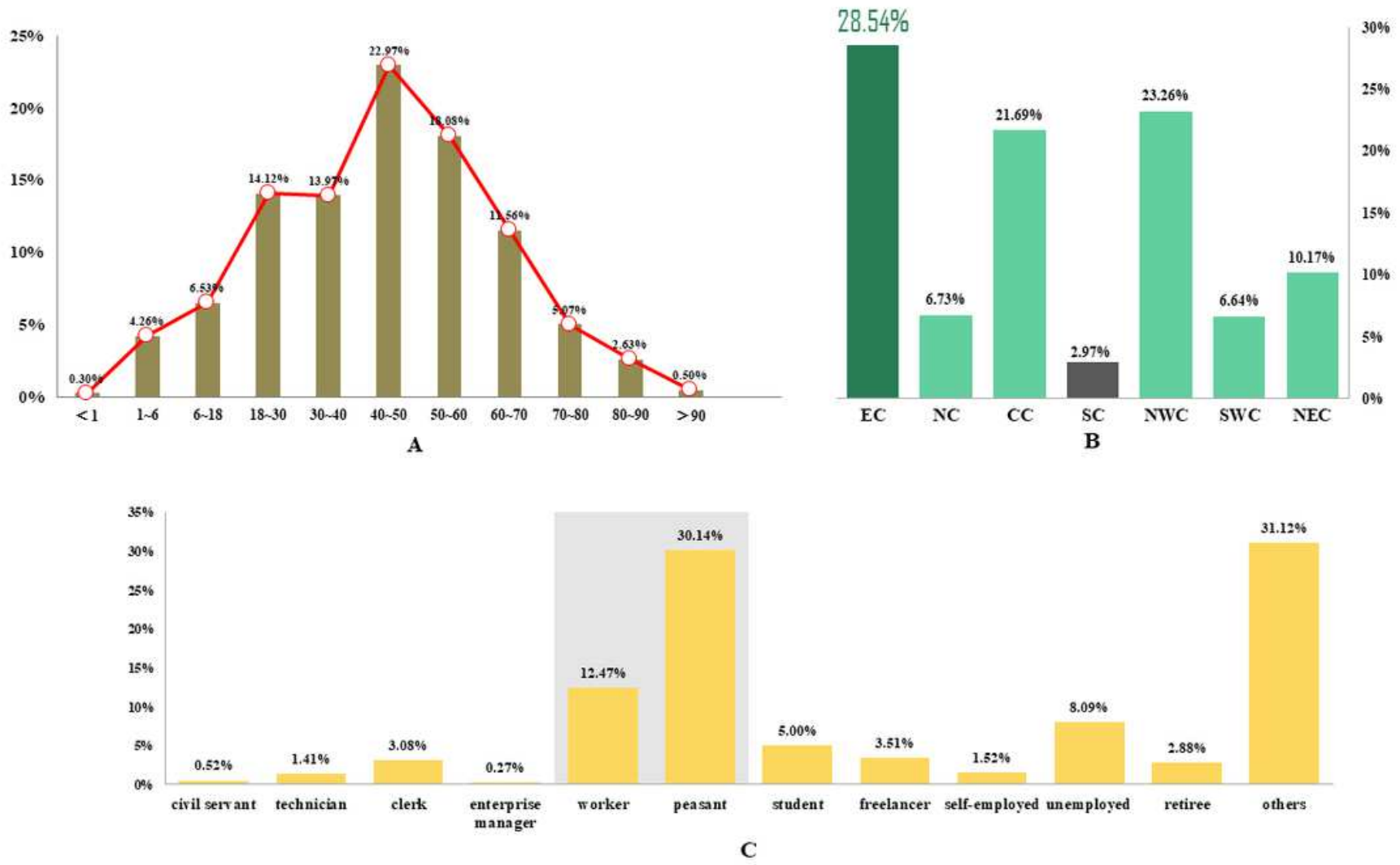

\section{Figure 1}

There were 62709 patients who were diagnosed with DTSI, of whom $67.41 \%$ were males with a mean age of $43.01 \pm 19.70$. Demographics are presented 


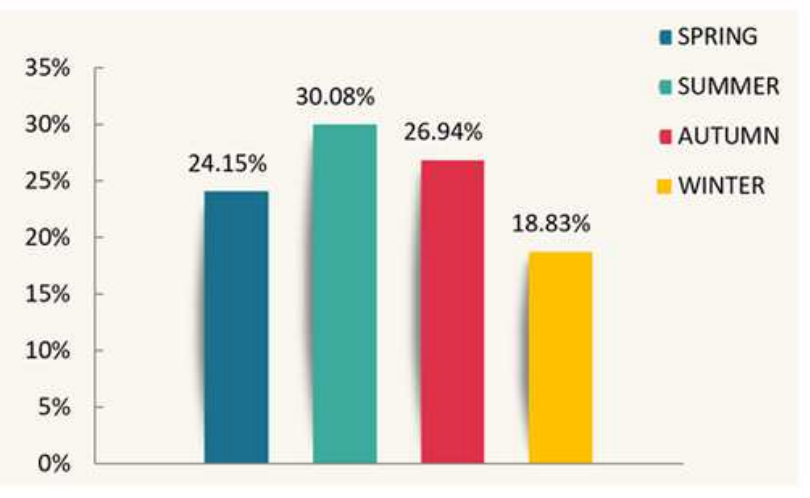

A

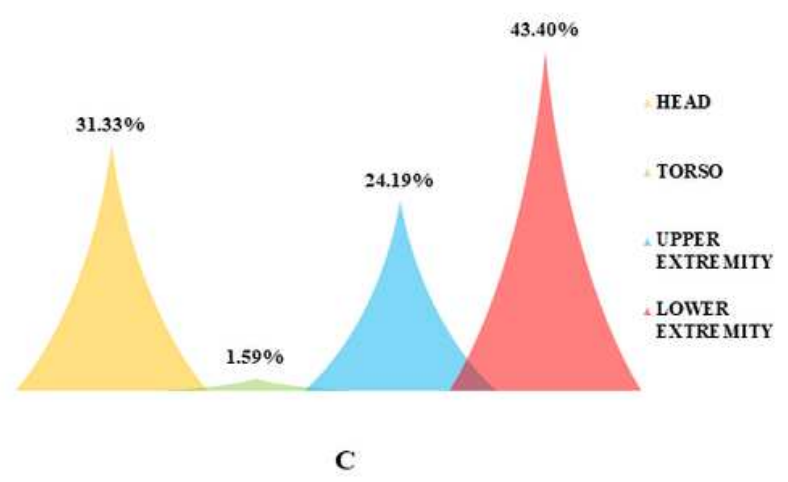

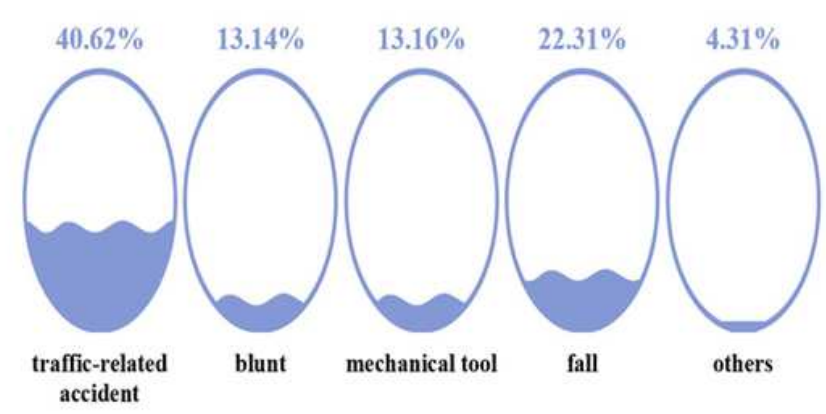

B

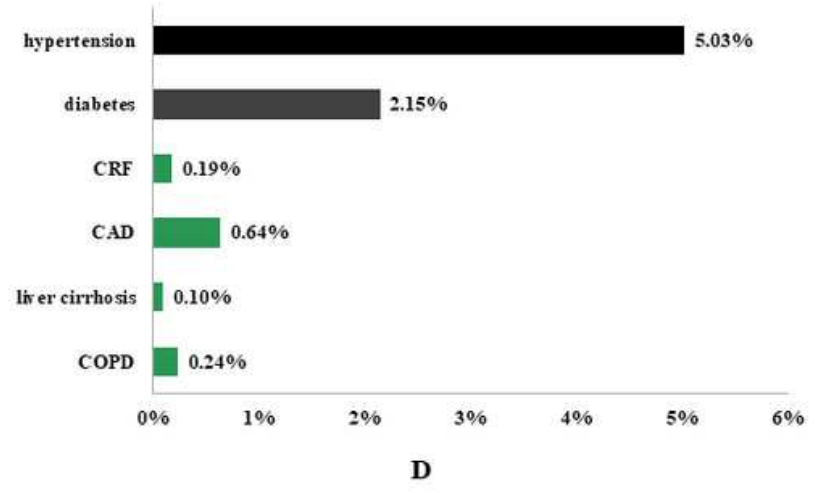

\section{Figure 2}

Patients with DSTI were hospitalized through emergency (58.79\%), and tended to increase in summer and autumn (Fig 2.A), with the majority concentrated in July and August (20.91\%). Traffic-related accidents was the leading mechanism of injury (40.62\%), followed by falls (22.31\%) (Fig 2.B). According to the diagnostic codes from ICD coding system, DSTI of different anatomic sites were classified into four groups, namely, head (DSTI of the scalp), torso (DSTI of the lower back), upper extremity (including DSTI of the upper arms, forearms and hands) and lower extremity (including DSTI of the thighs, calves and feet). The most frequent anatomic site of DSTI was lower extremity (43.40\%), followed by head (31.33\%), upper extremity (24.19\%) and torso (1.59\%) (Fig 2.C). Comorbidities were found in $8.34 \%$ of all the inpatients, with hypertension being the most common (5.03\%), followed by diabetes (2.15\%)(Fig 2.D). 
A

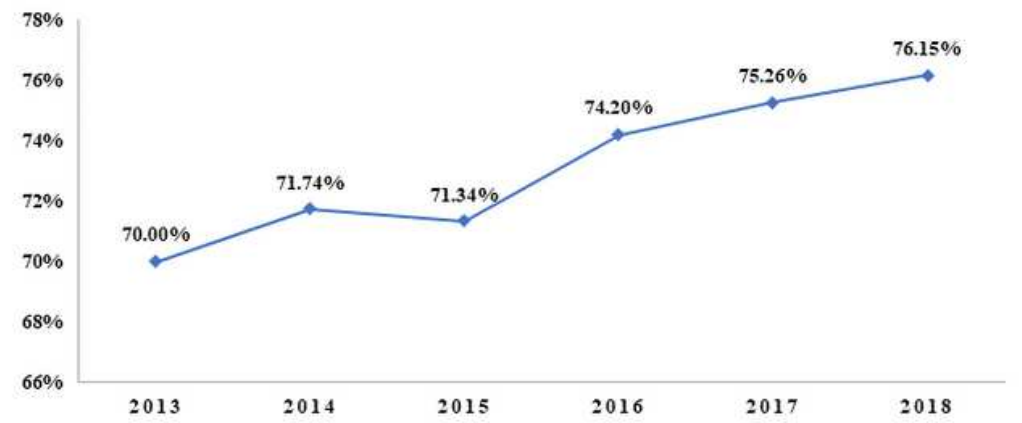

B

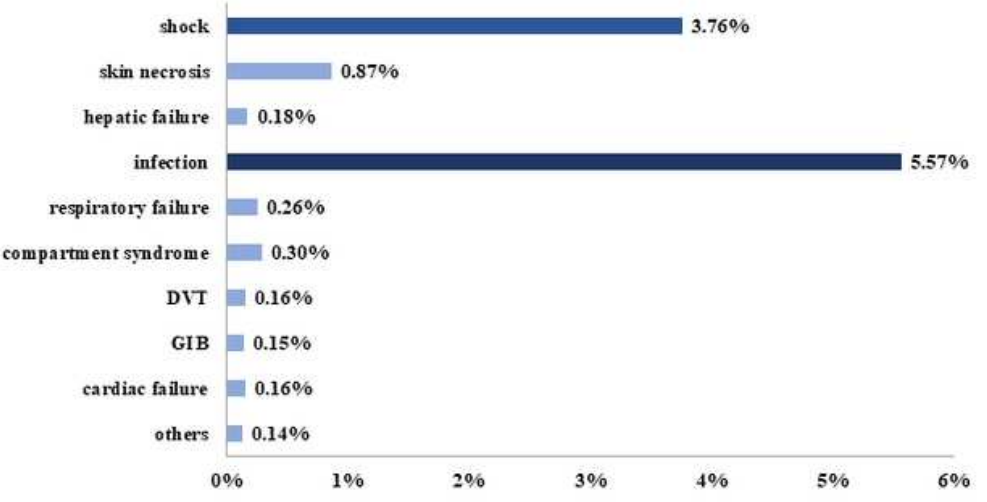

\section{Figure 3}

The operation rate of DSTI roughly showed a growing trend year by year (Fig 3.A), with a mean of $73.30 \%$, of which $37.09 \%$ were related to skin or flap transplanting and $31.93 \%$ underwent multi-operations. The average length of stay was $22.02 \pm 29.73$ days, during which time $0.93 \%$ of the patients ended up in death, and $12.13 \%$ developed complications, including infection (5.57\%) and shock (3.76\%), which happened far more often than the others (Fig 3.B). 


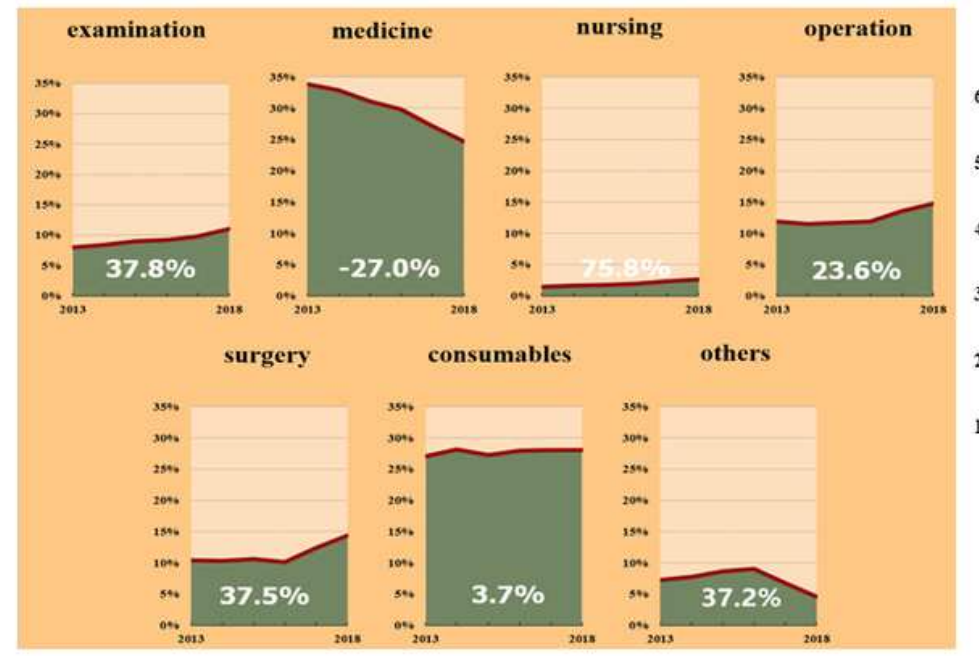

$\mathbf{A}$

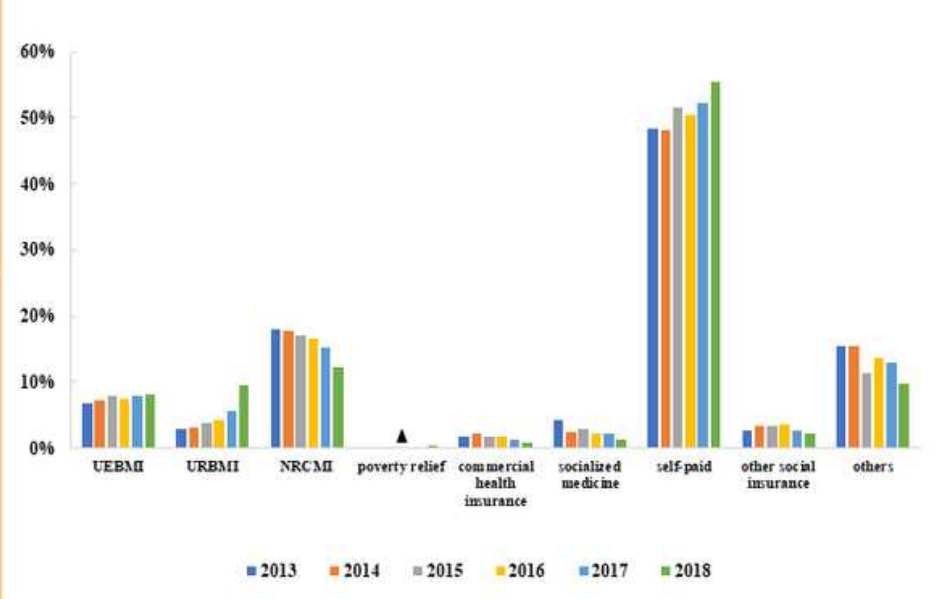

B

\section{Figure 4}

The average hospitalization expense of DSTI patients was 33.51 \pm 53.59 thousand RMB yuan. Medicine and consumables accounted for the largest proportion (59.57\%), but the former was decreasing markedly year by year, while growing trends emerged to varying degrees respectively among the rates of surgery, operation, nursing and examination expenses (Fig 4.A). More than half of the patients paid at their own charge $(51.10 \%)$, which proportion was rising together with that from by urban medical insurance, and those supported by the New Rural Cooperative Medical System had gradually decreased in recent years (Fig 4.B). 